\title{
Radio-over-Fibre access for sustainable Digital Cities
}

\author{
Trevor J. Hall • Ramón Maldonado-Basilio • Sawsan Abdul-Majid • \\ Joe Seregelyi · Ran Li · Irene Antolín-Pérez • Hamdam Nikkhah • \\ Frédéric Lucarz • Jean-Louis de Bougrenet de La Tocnaye • \\ Bruno Fracasso - Patrice Pajusco - Camilla Kärnfelt - Daniel Bourreau • \\ Michel Ney · Rabiaa Guemri · Yves Josse · Hexin Liu
}

Published online: 16 January 2013

(C) The Author(s) 2013. This article is published with open access at Springerlink.com

\begin{abstract}
Pervasive broadband access will transform cities to the net social, environmental and economic benefit of the e-City dweller as did the introduction of utility and transport network infrastructures. Yet without action, the quantity of greenhouse gas emissions attributable to the increasing energy consumption of access networks will become a serious threat to the environment. This paper introduces the vision of a 'sustainable Digital City' and then considers strategies to overcome economic and technical hurdles faced by engineers responsible for developing the information and communications technology (ICT) network infrastructure of a Digital City. In particular, ICT energy consumption, already an issue from an operating cost perspective, is responsible for $3 \%$ of global energy consumption and is growing unsustainably. A grand challenge is to conceive of networks, systems and devices that together can cap wireless network energy consumption whilst accommodating growth in the number of subscribers and the bandwidth of services. This paper provides some first research directions to tackle this grand challenge. A
\end{abstract}

T. J. Hall $(\bowtie) \cdot$ R. Maldonado-Basilio $\cdot$ S. Abdul-Majid ·

J. Seregelyi · R. Li · I. Antolín-Pérez · H. Nikkhah

Centre for Research in Photonics,

School of Electrical Engineering and Computer Science,

University of Ottawa, 800 King Edward Avenue,

Ottawa, Ontario K1N 6N5, Canada

e-mail: tjhall@uottawa.ca,Trevor.Hall@uottawa.ca

F. Lucarz - J.-L. de Bougrenet de La Tocnaye .

B. Fracasso - P. Pajusco - C. Kärnfelt - D. Bourreau •

M. Ney · R. Guemri · Y. Josse · H. Liu

Telecom Bretagne, Technopole Brest-Iroise,

CS 83818, 29238 Brest Cedex 3, France

F. Lucarz

e-mail: Frederic.Lucarz@telecom-bretagne.eu distributed antenna system with radio frequency (RF) transport over an optical fibre (or optical wireless in benign environments) distribution network is identified as best suited to wireless access in cluttered urban environments expected in a Digital City from an energy consumption perspective. This is a similar architecture to Radio-over-Fibre which, for decades, has been synonymous with RF transport over analogue intensity-modulated direct detection optical links. However, it is suggested herein that digital coherent optical transport of RF holds greater promise than the orthodox approach. The composition of the wireless and optical channels is then linear, which eases the digital signal processing tasks and permits robust wireless protocols to be used end-to-end natively which offers gains in terms of capacity and energy efficiency. The arguments are supported by simulation studies of distributed antenna systems and digital coherent Radio-over-Fibre links.

Keywords Radio-over-Fibre · Green ICT · Digital Cities

\section{Introduction}

Fixed wireless local area networks (WLAN) appeared in scene in the late 1990s for providing narrow-band wireless communication services in public places, for instance, in airport terminals. Their rapid proliferation has reached private (home) services, becoming available through digital subscriber lines and cable access modems, hence providing fixed wireless Internet services to subscribers [1]. A distinctive feature of such WLAN networks is their 'narrow band' services, offering transmission rates up to $54 \mathrm{Mbps}$ with carrier frequencies in the $2.4 \mathrm{GHz}$ (standard IEEE $802.11 \mathrm{a} / \mathrm{g}$ ) and $5 \mathrm{GHz}$ (HiperLAN2) bands. The explosive growth of the Internet and the consequent demand for data, 
voice and video applications has brought about the concept of ubiquitous connectivity (communication anytime, anywhere and with anything). As a consequence, today's wireless local networks must meet consumer demands to provide an increased transmission capacity while maintaining sufficient coverage [2-5]. The capacity can be increased by using carriers at higher frequencies (for instance, the use of the unlicensed millimetre frequencies at around $60 \mathrm{GHz}$ ), which also results in reduced reach and, hence, service areas (cells) of reduced size [6]. A smaller cell size implies a larger number of remote antenna units (RAU), and therefore, extensive feeder networks are required to provide the demanded service. In the end, the cost of the RAUs (which in this case is mainly determined by the frequency of the RF carrier), the feeder network and the system installation and maintenance, in combination with the demanded broadband services (multi-gigabit per second (Gbps)), have brought to the scene the appearance of new paradigms in broadband wireless access networks.

Whereas the majority of efforts by both the industry and the research community have been focused on the technical and technological aspects of improving the quality and speed of the telecommunication services of the present WLANs (and creating new network paradigms), the ecological and environmental aspects in terms of energy efficiency/savings have taken second place. The objective of this manuscript is twofold. From the environmental perspective, it presents an open discussion of the sustainability aspects that should be considered in wireless access networks towards the conception of a Digital City of the future. From the technical point of view, it addresses the application of distributed antenna systems as a feasible approach to reduce the energy consumption in the wireless access points of present and future broadband WLANs. Nevertheless, the Radio-over-Fibre (RoF) network paradigm as a feasible approach to provide broadband wireless services to end users is also introduced and discussed. The Radioover-Fibre networks are analysed within the frame of optical coherent systems, thus providing technical and environmental advantages relative to the traditional analogue systems making use of intensity modulation and direct detection techniques.

The manuscript is organised as follows. Section 2 describes the envisaged context (use case scenario) for wireless access technology. It introduces the concept of wireless/ cloud computing/ambient intelligence as an 'intelligent urban network infrastructure' that will shape cities of the future. It raises sustainability issues that have been overlooked by urban ecologists but which are of paramount importance to engineers responsible for creating this infrastructure. It emphasises that a whole system perspective is necessary and that such approaches may have nonintuitive and different outcomes than a simple minded focus on 'energy efficiency'. Section 3 raises the issue of energy consumption of wireless access networks as a particular issue in the proposed scenario (Digital Cities) and identifies the fundamental problem as the wide angle radiation patterns of wireless (assuming the profligacy elsewhere is removed by good engineering). Distributed antenna systems (DAS) are found to be the answer in terms of coverage and total power in a cluttered urban environment, providing an incremental advance of directionality from simple powering down of excess capacity to advanced beam-forming systems, e.g. multiple-input multiple-output systems. Numerical simulations based on a simple model of electromagnetic waves propagation are implemented to demonstrate the concept of energy efficiency in wireless access networks operating at $2.4 \mathrm{GHz}$ and millimetre-waves. Motivated by the perspective of energy consumption in a Digital City scenario rather than the usual argument about cost (also important), the paper arrives at a Digital Coherent Radio-over-Fibre architecture described in Section 4. The argument for a fibre optic feeder network (a Digital City may be expected to be fibrerich) rests on its established energy efficiency compared to copper or microwave backhaul (optical wireless backhaul and optical wireless access are options in benign environments). The digital coherent RoF networks can outperform analogue intensity-modulated direct detection systems from an energy consumption perspective and, important enough, in the quality and the bandwidth of the telecommunication services. Numerical simulations on optimised digital coherent Radio-over-Fibre systems at millimetre-wave carriers have been implemented. The optimisation is implemented in terms of the photonic generation of the carrier signals, as opposed to their generation by electronic approaches. Nevertheless, the coherent system offers the advantage of an optimised component counts in an architecture free of hungry energy consumers such as Erbium-doped fibre amplifiers. A significant outcome of this section is the identification of the RF to optical single sideband upconverter (mixer) as the weakest link of the digital RoF architecture. Research is required to improve power consumption, conversion efficiency, carrier and sideband suppression, footprint and stability (integration without a need for trimming). At the end of the manuscript, conclusions and take-home messages are presented.

\section{Sustainable Digital Cities}

\subsection{Intelligent network urban infrastructure}

As of 2008 , over $50 \%$ of the world's population live in cities, and this number is expected to rise to $80 \%$ by 
2020 and reach 6.4 billion by 2050 [7]. For instance, in the province of Ontario (Canada), urban areas already attract $85 \%$ of the population [8]. Cities have become the major economic units in the global economy and are responsible for the bulk of innovation. The structure of cities has been radically transformed by the introduction of utility, transport and now information and communications technology (ICT) network infrastructure to propel cities into the digital age and e-economy.

Urban planners envisage a Digital City in which ICT enables all operations to be more efficient and effective. To that purpose, it is assumed that ambient intelligence is embedded in the fabric of the city, wherein various sensors are widely and densely deployed to gather all sorts of information (traffic, pollution, temperature, etc.) which is then transported by wireless and wired access networks to data centres for analysis in order to optimise the use of resources. Ubiquitous wireless broadband and computing access provides all imaginable services to citizens, enterprises and public services, and enables citizens to be well integrated into the life of their community and the city. Our vision is of an information-rich, economically efficient and effective, city that is clean, healthy and aesthetically pleasing.

The socioeconomic benefits of Digital Cities are subject to vast literature (see URENIO Intelligent Cities [9]). The results of Digital City research are amenable to commercialisation over both short and long terms. Digital Cities are now big business for some major companies-as seen on the websites of IBM (Smarter Cities [10]), Siemens (City of the Future [11]) or Philips (Liveable Cities [12])—and commercial interest extends well beyond the electronics industry to include, for example, municipalities, heavy engineering companies and urban furniture manufacturers.

\subsection{Sustainability and ICT}

\subsubsection{Green by ICT}

Climate change has alerted society to the urgent need to reduce greenhouse gas (GHG) emissions. In that regard, ICT networks can substantially lower GHG (Green by $I C T$ ) by facilitating remote interactions (tele-working, e-conferencing, e-learning, e-health monitoring) and by effective use of resources (simplified supply chain management, lower cost lighting, heating, computing, etc.). For instance, EUROCITIES, a coalition of more than 140 large cities in over 30 European countries, has set up a framework based on the exploitation of ICT to develop greener cities. An ongoing European project NiCE [13] focusing on energy efficiency in intelligent cities supports the Green Digital Charter with an aim to use ICT to significantly reduce energy consumption and $\mathrm{CO}_{2}$ emissions.

\subsubsection{Growing concerns on the environmental impact of ICT}

However, globally, about $80 \%$ of the electricity consumed is generated by burning carbon-based fuels [14], and this proportion is unlikely to change greatly in the foreseeable future. Besides, ICT has been ranked as the fifth largest industry in terms of electrical power consumption corresponding to $2-6 \%$ of the total energy consumed worldwide [15]. As an example, Telecom Italia is reported as the second largest consumer of electrical power in Italy after the railway system $[16,17]$. In the European Union, recent statistics [18] revealed that ICT equipment and services consumed over $8 \%$ of electrical power and produced about $4 \%$ of its $\mathrm{CO}_{2}$ emissions in 2010.

Increased use of ICT is outstripping energy efficiency improvements, leading to unsustainable growth in energy consumption, faster than any other industry sector; ICT GHG is currently doubling every 4 years [19]. With projected growth in traffic, the provision of a good proportion of the world's population with equal access to broadband services, machine-to-machine communication outstripping human-to-machine communication, a time is inevitably reached when energy consumption, GHG emissions and operating temperatures exceed reasonable bounds. Estimates vary, but the 'business as usual' approach will be unsustainable within a few decades [20].

\subsubsection{The search for solutions}

Can we look to renewable energy for zero GHG emission solutions to the increasing demand by ICT for electricity? Urban areas could be provided with broadband services via optical communication links from remote data centres strategically positioned near sources of renewable energy. It is worth noting that network operators are investing in wind farms and other sources of renewable energy to offset part of their carbon footprint. This can be complemented by intelligent energy management that, amongst other whole system optimisations, opportunistically harvest renewable energy when available and offsets energy consumed by heating and cooling, making the best use of the waste heat from ICT infrastructure.

However, even if it were possible to supply all the energy needed from renewable sources, there would remain economic and technical roadblocks to the scaling up of ICT networks. Without other measures, energy consumption would eventually outstrip supply and heat dissipation would become unmanageable. For example, it has been estimated that, with current growth in demand, core IP routers must operate at temperatures found at the surface of the sun within a couple of decades [20]. Therefore, more radical measures are required to fully address societal concerns 
regarding sustainable development and potential health risks associated with increased RF exposure. These radical measures will entail new engineering criteria and rules and, ultimately, new ultra-low-energy component technology aimed at reducing overall energy consumption and GHG emissions.

\subsubsection{Why energy efficiency is not enough}

As huge consumers of electrical energy, network providers have shown strong determination over the past 3 years to improve energy efficiency. In the context of current telecommunication networks, energy efficiency is conveniently expressed as the amount of energy (in Joules) that is required to move an array of data (in bits) across a device or a communication link. OVUM has published two reports: Surfing the Green Wave in Telecom [21] in 2008 and Increased Focus on Network Power Consumption to Lower OpEx, Go Green [22] in 2009.

In "Surfing the Green Wave in Telecom", OVUM advises: "Going green must serve the overriding goal of making money for their shareholders. As such three realities should drive action. First, energy-electricity, mainlyhas significant direct costs for telecos, and these costs are rising, in some cases dramatically. Second, environmental and energy cost concerns give rise to a number of sizable business opportunities to service providers. Third, green is 'in': credible, sustained commitments by corporations to environmentally friendly practices can pay off with public goodwill."

While 'green' thus remains a secondary priority of business strategies, it is at least a start that operating cost reduction in the form of improved energy efficiency is being branded as green. However, energy efficiency alone is not enough for a truly green ICT. Whilst welcome, an exclusive focus by operators on energy efficiency is not sufficient to ensure sustainability and may in fact cause net GHG emissions to increase for the following reasons:

1. Increased energy efficiency paradoxically leads to increased energy consumption (Jevon's paradox, also known as the Khazzoom-Brookes postulate [23]). In essence, the increased energy efficiency encourages an even greater increase in use. It is clear that mobile wireless follows this economic paradox.

2. Improvements in energy efficiency in one part of a complex system could lead to increased energy consumption in another part of the system.

For example, a study carried out by the International Institute for Sustainable Development [24] and funded by Canada's Advanced Research and Innovation Network (CANARIE) [25] assessed the business case for moving University data centres to remote, zero-carbon (i.e. powered with renewable energy) facilities. While the relocated data centres are powered by sources of substantial renewable energy, the computing services are delivered over broadband (optical) networks, c.f. 'cloud computing'. ICT accounts for a significant but largely unquantified proportion of a university's electrical consumption. In the case of the University of Ottawa, it was found that a business case for relocation did not exist because the university successfully converts waste energy from ICT into energy needed elsewhere on campus to heat and cool buildings and to provide hot water. This nonintuitive result amply illustrates the need for a whole systems (including socioeconomic factors) approach to the optimization of energy management. The report also illustrated the need for improvements in the granularity of consumption data logging to ensure adequate information for analysis. In the ideal sustainable Digital City, one might envisage wireless sensor networks logging at the granularity of individual outlets.

\subsubsection{The case of mobile and wireless access}

With the worldwide adoption of wireless solutions, mobile and wireless access networks now act as a major contributor to ICT energy consumption and $\mathrm{CO}_{2}$ emissions. From a network operator perspective, energy consumption dedicated to mobile network operation is on the rise and becomes alarming in view of subscriber/user growth predictions. For instance, the total energy consumption of the France Telecom network was estimated in 2006 at $1,341.5 \mathrm{GW}$ h/year of which $21 \%$ is accounted for by the mobile wireless network (57\% of this is attributable to wireless base stations) [26]. These statistics are representative of most major network operators worldwide.

Amongst all access technologies, RF wireless remains one of the most power-hungry means of communication. For instance, wireless access using WiMAX can be up to ten times less energy-efficient than photonics-based wired solutions based on passive optical networks (PON) for access speeds ranging from $1 \mathrm{Mb} / \mathrm{s}$ to $1 \mathrm{~Gb} / \mathrm{s}$ [27]. Yet, the sheer convenience of an untethered wireless connection guarantees growth in subscriber numbers. Wireless mesh networks are being deployed extensively in dense urban areas not only for in-building access (train stations, shopping malls, stadiums, offices, homes) but also for outdoor access in streets and highways. It is foreseen that ultimately, all final links will be wireless, even if the distance spanned is as little as a metre. Wireless access points at $60 \mathrm{GHz}$ may well be deployed every $10 \mathrm{~m}$ in a Digital City use case scenario [28].

Growth in bandwidth demand also continues unabated, driven by new services and applications; in order to avoid a bottleneck, the wireless link must have commensurate capacity growth. The energy consumed by mobile 
and wireless access networks and their contribution to GHG emissions are therefore set to increase with capacity growth.

The need for a paradigm shift in network design has been recognised by the recent formation of an AlcatelLucent led consortium of industry and academia named Green Touch ${ }^{\mathrm{TM}}$ whose goal is to create technologies that will make communication networks a thousand times more energy-efficient [29] than they are now. Ongoing EUfunded projects EARTH [30], OPERANet I [31] and II [32], and TREND NoE [33] address energy efficiency of mobile/ wireless access networks. Fortunately, current practice is profligate in respect of energy consumption, and many energy savings can be made from power-efficient and energy-aware protocols and architectures (e.g. sleep mode, circuit vs. packet switching) to low-energy consumption implementations (e.g. replacement of copper or wireless access by photonics-based solutions) and energy harvesting from the environment (e.g. solar cells). Addressing energy consumption at the physical layer is a mandatory research objective to complement the aforementioned endeavors.

\section{Distributed antenna systems}

\subsection{Fundamental issue of RF wireless}

One fundamental issue of RF wireless is its broadcast nature compounded by the inefficiency of antennas that are small compared to the wavelength - the transmitted power is radiated in broad beams of which only a minute fraction is intercepted by the receiver.

However, in practice, the energy consumption of mobile terminals in operation is negligible in comparison to that of base stations (a ratio of 1:150 [34]). This is a testament to the great strides that have been made in reducing the power consumption of mobile terminals enabled by application-specific integrated circuits (ASIC) and driven by the requirement to minimise the bulk of the terminal and to extend battery life, rather than by any concern for the environment. The concern to reduce energy consumption has not previously been applied to the design of base stations: their lower volumes do not warrant ASIC technology, and the alternative is power-hungry field programmable gate arrays.

The total power consumption of a base station varies proportionally to the usefully radiated RF power even though the latter is only 5-10\% of the total. The RF power amplifiers are approximately $45 \%$ efficient, contributing a further $11-22 \%$. Fans account for $10-15 \%$, while air conditioning accounts for $50 \%$ of the total consumed power. The signal processing and switching hardware consume power proportional to the base station data capacity, which also scales with RF power, either by capturing more mobile handsets or by providing higher data rate links to end users. If the link between the transmitter and the receiver could be made more directional, the transmitted power could be reduced and, with it all other components of base station energy consumption. A major milestone would be the achievement of uncooled operation.

\subsection{Towards a higher degree of densification in wireless access networks}

The ever-growing demand for broadband services with enhanced capacity and mobility is a significant factor in driving mobile and wireless access networks towards a higher degree of densification. The implementation of distributed antenna systems (DAS) is considered a good solution to meet this demand in the present context of Digital Cities. Indeed, such systems are already known to improve not only coverage and performance of wireless communications in indoor environments [35] but could also lead to better energy efficiency in mobile and wireless networks especially when operating at millimetre-wave (mm-wave) frequencies.

It turns out that the total RF power for the same coverage is smaller in the distributed case, provided that the exponent of the propagation model is greater than two [36], which is generally the case in cluttered urban or indoor environments. This can be shown using a rather simple RF propagation model as described below. The received power $\mathrm{P}_{R_{x}}$ at the wireless terminal can be computed according to the large scale characterization of the channel model [37] using the following equations:

$$
\begin{aligned}
& P_{R_{x}}(\mathrm{dBm})=P_{T_{x}}+G_{T_{x}}+G_{R_{x}}-\mathrm{PL}-X_{\sigma} \\
& \mathrm{PL}(\mathrm{dB})=\mathrm{PL}\left(d_{0}\right)+10 n \log _{10}\left(\frac{d}{d_{0}}\right)_{\sigma}
\end{aligned}
$$

with $P_{R_{x}}$, received power; $P_{T_{x}}$, transmit power; $G_{T_{x}}$, transmitter antenna gain; $G_{R_{x}}$, receiver antenna gain; PL, path loss term; $X_{\sigma}$, the shadowing term; and $n$, path loss exponent. In this simple model, the path loss $\operatorname{PL}\left(d_{0}\right)$ and a power decay index $n$ are assumed according to the carrier frequency under study. The broad predictions of the model described in Eqs. 1 and 2 are supported by simulations of propagation by using more sophisticated planning tools. Results are presented in the following subsections.

\subsubsection{DAS in 2.4 GHz wireless access}

Figure 1 shows the advantage of using distributed antennas rather than a single antenna to provide wireless coverage at $2.45 \mathrm{GHz}$ in an indoor environment, by illustrating the received power level in decibels relative 


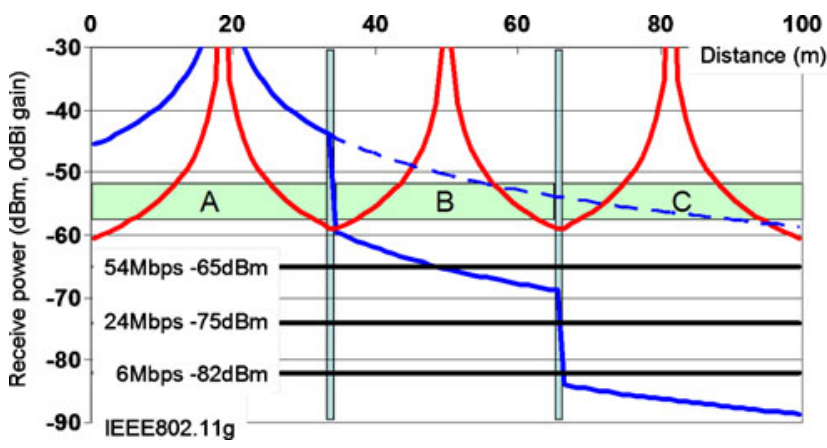

Fig. 1 Received power (in decibel-milliwatt) with distance (in metre) in the case of distributed antennas vs. single antenna systems

to one milliwatt $(\mathrm{dBm})$ as a function of distance in both cases. In the present example, the indoor environment comprises two walls separated by $32 \mathrm{~m}$ from each other to form three zones referenced as $\mathrm{A}, \mathrm{B}$ and $\mathrm{C}$ in Fig. 1. The Wi-Fi receiver sensitivity levels (in decibelmilliwatt) required for different nominal bit rates according to the IEEE $802.11 \mathrm{~g}$ standard are also represented in Fig. 1 .

Using a single omnidirectional antenna (0 dBi gain) with a total transmit power of $20 \mathrm{dBm}$ (equivalent isotropic radiated power, EIRP), the power obtained at the receiver side suffers from a 15-dB attenuation each time it traverses a wall, as illustrated by the solid blue line in Fig. 1. The 15-dB attenuation was measured in a laboratory at Télécom Bretagne, considering vertically polarised waves hitting the surface of the walls at an incident angle of $45^{\circ}$. The dashed blue line depicts the power decay due to free space losses simulated without the presence of walls.

With the use of three omnidirectional antennas (each of them having a $5-\mathrm{dBm}$ EIRP), the maximum data rate (54 Mbps) can now be reached in each of the three zones (A, B and C) as shown by the combined power in the red trace. In contrast, the maximum data rate in the single antenna case can be reached only in zone A and partly in zone B. This demonstrates that distributed antennas drastically improve the overall wireless coverage. Besides, the total transmit power (three antennas with 5-dBm EIRP each) is reduced by $10 \mathrm{~dB}$ as compared with the single antenna case, thereby reducing the overall energy consumption. Further energy savings may be achieved through innovative circuit design and network strategies for turning off antenna units whenever and wherever transmission resources are not required by user terminals, which in turns mitigates human exposure and electromagnetic interference problems.

In order to estimate the real benefits of DAS, appropriate energy efficiency metrics should be defined, taking both power consumption and performance into account. To this end, the energy-per-bit goodput (EBG) can be used [38], indicating how much energy the system has to spend to transmit one bit of payload data. As an illustration [39], an EBG simulation of a DAS system operating under the standard IEEE 802.11 and using Radio-over-Fibre links is shown in Fig. 2. The indoor building floor area is $40 \mathrm{~m} \times 40 \mathrm{~m}$, and a power decay index of $n=5.1$ is taken as a path loss parameter.

The mean computed EBG in the centralised antenna system (Fig. 2a) is $1.35 \mathrm{~J} / \mathrm{Mbit}$ and the value decreases to $0.357 \mathrm{~J} / \mathrm{Mbit}$ for the four antenna DAS configuration of Fig. 2b. Despite additional power consumption at the optoelectronic interfaces and the decrease in the overall radiated power by a factor of two, the energy efficiency is 3.8 times better in the distributed antenna case (Fig. 2b). Considering omnidirectional antennas and the characteristic impedance of vacuum, the mean and the maximum strengths of the electric field received in the area are computed in Table 1. When the users are close to the centralised antenna, the maximum strength of the electrical field is three times higher than in the DAS case.

A system demonstrator was set up on the CapilR $^{\mathrm{TM}}$ platform at Télécom Bretagne to provide enhanced $2.4 \mathrm{GHz}$ Wi-Fi coverage within an office building (ARAGO Centre) by means of four distributed omnidirectional antennas (Hyperlink model HG2458CU). The antennas are positioned in a way to obtain the best possible coverage in the office spaces. The simulation tool WinProp ${ }^{\mathrm{TM}}$ was used with its module WallMan ${ }^{\mathrm{TM}}$ to model the office building, taking into account the frequency dependence of the material properties (transmission and reflection losses). The simulation was done in the ProMan ${ }^{\mathrm{TM}}$ module using the dominant path method and the wireless norm IEEE802.11g. Simulation results were confirmed by measurement campaigns. In the DAS case, the Wi-Fi box (Buffalo Wireless router) was set to transmit $1 \mathrm{dBm}$, which, after distribution through RF cables and power splitters, meant an EIRP at each antenna of $-17 \mathrm{dBm}(0.02 \mathrm{~mW})$. To achieve the same coverage and bit rate with a single antenna system (centred antenna), the transmitted power from the Wi-Fi box had to be increased to $10 \mathrm{dBm}(10 \mathrm{~mW})$, which resulted in $-2 \mathrm{dBm}(0.63 \mathrm{~mW})$ EIRP at the antenna after taking into account the cable losses. Figure 3 illustrates the received power distribution within an office building, for both a DAS with four antennas and a conventional single antenna system. Both systems provide equal service to the end users in terms of bit rates. The simulation results, which are confirmed by measurements, showed that a $15 \mathrm{~dB}$ reduction in RF power exposure can be achieved by the DAS as compared with the single antenna system. In other words, users located closest to the antenna of the single antenna system are exposed to a $15-\mathrm{dB}$ higher radiated power than those being closest to any one of the antennas of the DAS. This confirms that DAS can be advantageously 
Fig. 2 (a) Energy-per-bit map for different IEEE 802.11 data rates with one access point, considering a radiated power of $17 \mathrm{dBm}$ and (b) the same computation with four access points delivering a total power radiation of $14 \mathrm{dBm}$
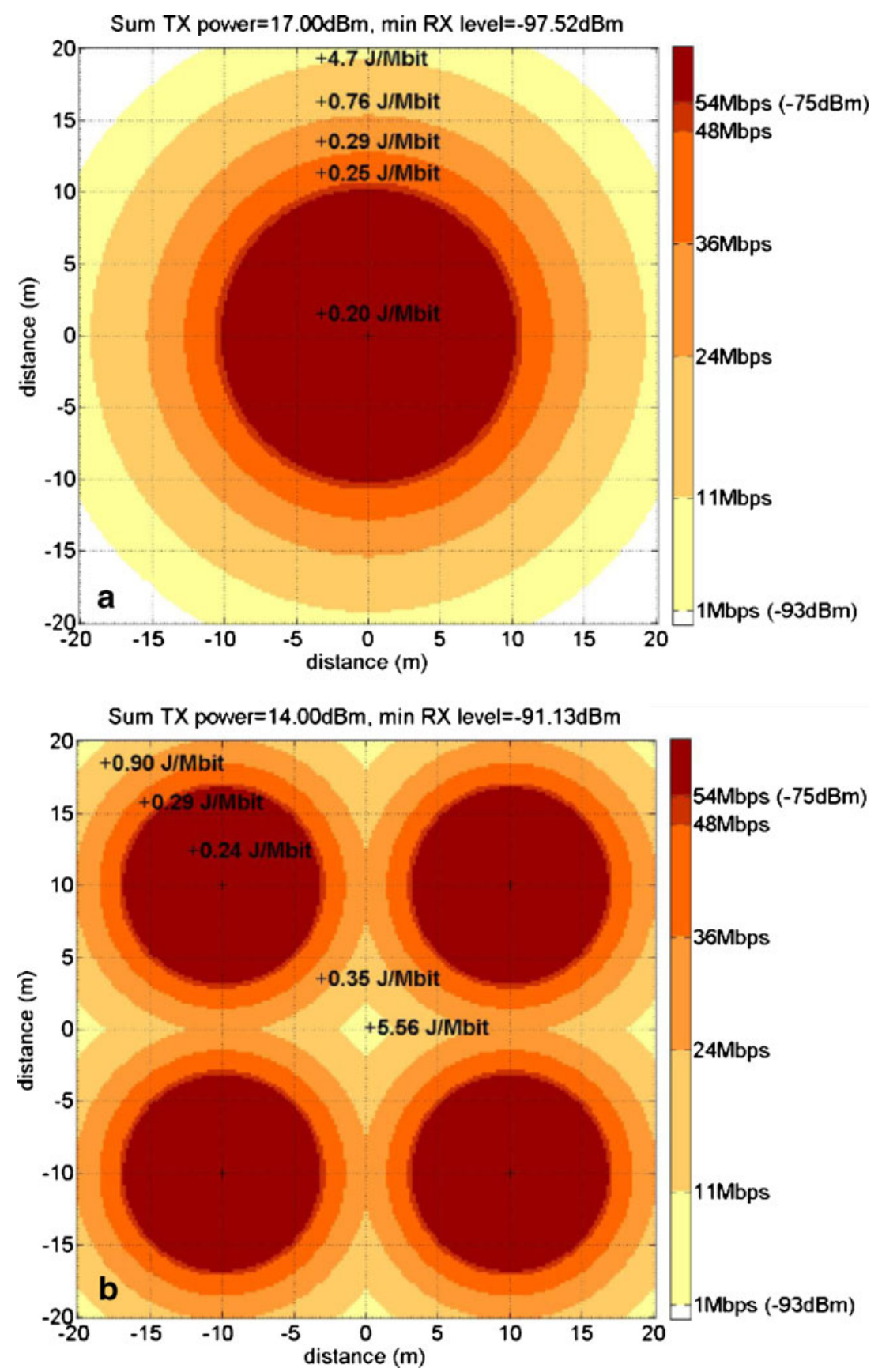

used for minimizing overall transmitted power, thus mitigating electromagnetic exposure levels and electromagnetic interference problems.

\subsubsection{DAS in $60 \mathrm{GHz}$ wireless access}

Most RF wireless technologies operate at microwave frequency ranges. However, a transition to mm-wave transmission frequencies (e.g. $60 \mathrm{GHz}$ ) is required to provide new bandwidth-hungry applications with large continuous blocks of RF spectrum. It also enables higher antenna gain from small antennas and offers compactness by keeping the overall dimensions of antenna arrays compatible with a handheld mobile terminal. The increased directionality and increased atmospheric absorption of $60-\mathrm{GHz}$ wireless links ( $\sim 15.5 \mathrm{~dB} / \mathrm{km}[40])$ also mandates shorter ranges. Such a densification entails deploying denser sets of smaller coverage cells, thereby increasing the overall number of remote antenna units (i.e. base stations or wireless access points) for a given surface area.

From a physical viewpoint, the high losses of $60-\mathrm{GHz}$ wireless links can be compensated by using high gain 
Table 1 Energy efficiency and RF exposure comparison between the centralised antenna and the DAS configurations as considered in Fig. 2

\begin{tabular}{lcc}
\hline Criteria & $\begin{array}{l}\text { Centralised } \\
\text { antenna }\end{array}$ & DAS \\
\hline Total radiated power & $17 \mathrm{dBm}$ & $14 \mathrm{dBm}$ \\
Mean throughput reachable & $23.8 \mathrm{Mbit} / \mathrm{s}$ & $37.4 \mathrm{Mbit} / \mathrm{s}$ \\
Mean goodput & $9.38 \mathrm{Mbit} / \mathrm{s}$ & $14.4 \mathrm{Mbit} / \mathrm{s}$ \\
Mean E & $1.35 \mathrm{~J} / \mathrm{Mbit}$ & $0.357 \mathrm{~J} / \mathrm{Mbit}$ \\
Max electrical field received & $1.20 \mathrm{~V} / \mathrm{m}$ & $0.427 \mathrm{~V} / \mathrm{m}$ \\
Mean electrical field received & $1.42 \mathrm{mV} / \mathrm{m}$ & $9.95 \mathrm{mV} / \mathrm{m}$ \\
\hline
\end{tabular}

antennas $(\sim 50 \mathrm{dBi})$ to extend propagation up to the kilometre range, which can be considered for point-to-point links in outdoor environments such as those envisaged in the Digital City scenario. From an economical viewpoint, low-cost components at mm-wave frequencies, including power amplifiers, are still difficult to obtain, even though 60-GHz RF-CMOS technology is expected to bring costs down. As described in an ongoing project Optical Radio Infrastructure for Gigabit Indoor Networks (ORIGIN, FUI8, 2009-2013), the search for solutions to further optimise wireless coverage on a room-by-room basis becomes a key issue while using low power amplifiers (i.e. 10-dBm output power).

Wireless access network architecture based on DAS benefits from the strategic positioning of the various antennas while adapting their radiating patterns according to their environment. In this context, low-cost $60-\mathrm{GHz}$ wideband horn antennas based on foam technology [41] are particularly attractive from an economical viewpoint. Indeed, their aperture dimension can be freely chosen (no constraint of standard dimensions) with the same low-cost manufacturing. As a result, they can provide a quasiconstant directivity if one keeps their effective aperture
Fig. 3 Received power (in decibels relative to one milliwatt) for a DAS system with four antennas (a) and with a single antenna (b) a

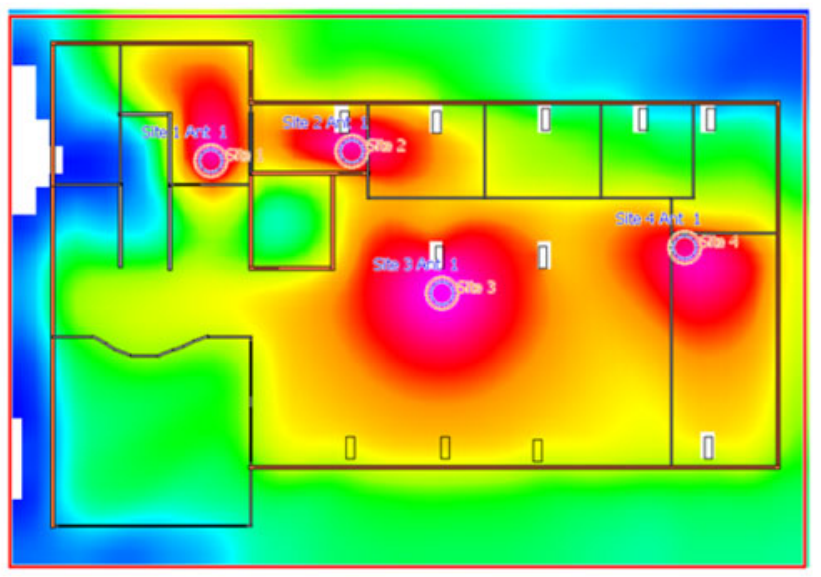

b

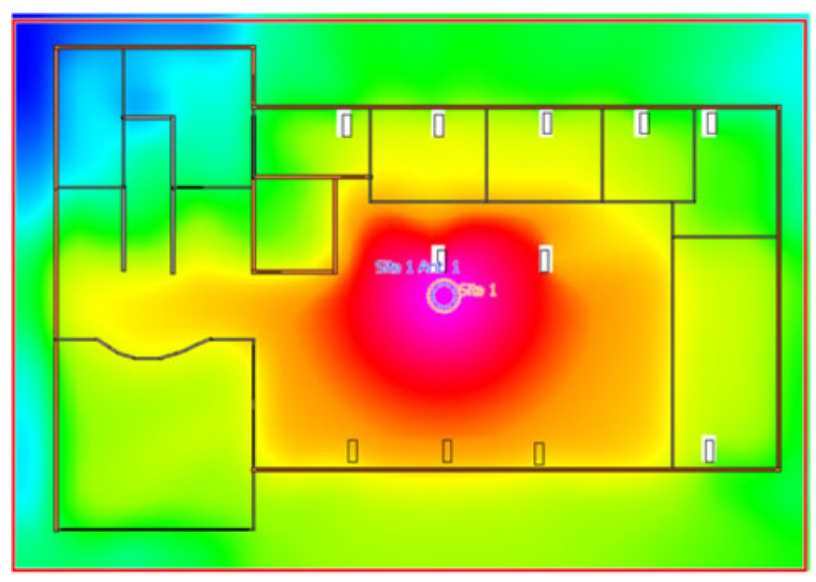

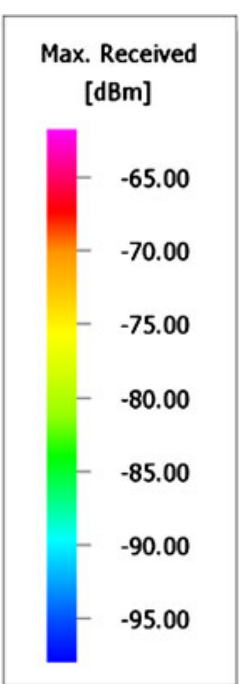

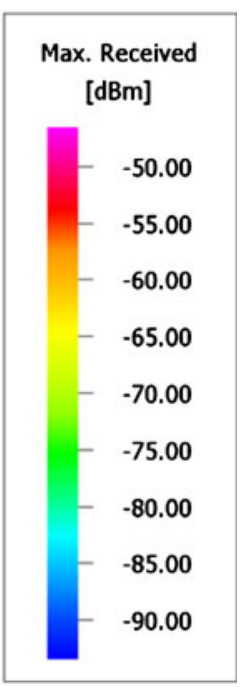


Fig. 4 Simulated wireless coverage (Pr level) of a 60-GHz foam antenna for different positions in the horizontal plane $(\mathrm{x}, \mathrm{y})$ and different values of the tilt angle (from the ceiling plane): $45^{\circ}$ (a), $25^{\circ}(\mathbf{b}), 30^{\circ}$ (c) (a) Tilt $=45^{\circ}$

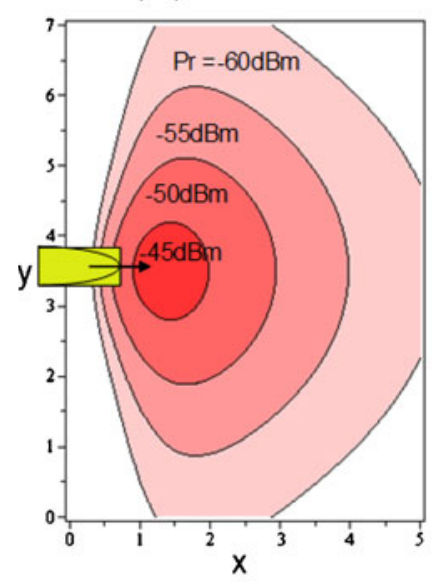

$\operatorname{Pr}=-60,-55,-50,-45 \mathrm{dBm}$ (b) Tilt $=25^{\circ}$
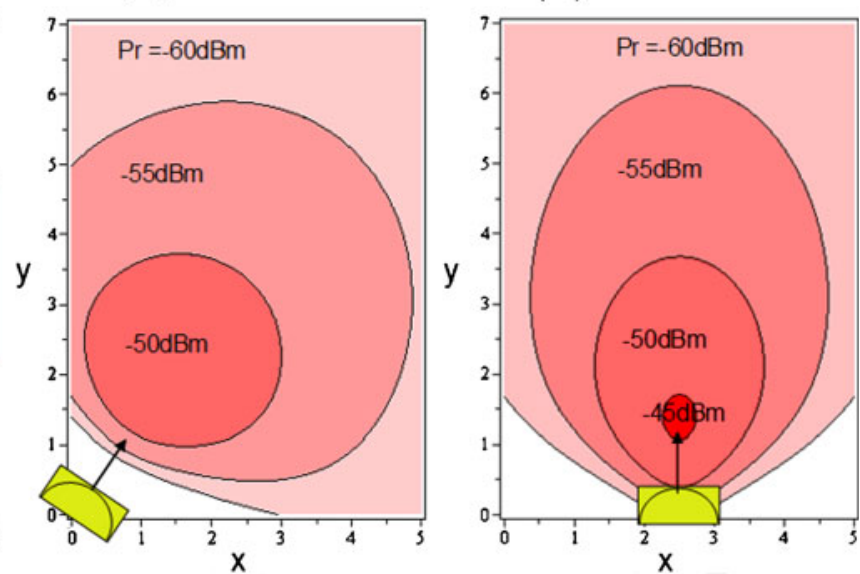

Dimension $x, y$ : metre area constant while adjusting the main beam width in both planes according to need. Simulations of the wireless coverage of a $60-\mathrm{GHz}$ foam antenna carried out at Télécom Bretagne show that it is possible to achieve quasi "line of sight" (LOS) propagation by using six-sector horn antennas, each having a measured gain of $10.3 \mathrm{dBi}$ and an EIRP of $20.3 \mathrm{dBm}$, combined with a $10 \mathrm{dBi}$ gain receiving antenna. Under LOS conditions and a given relative position between the transmitter and receiver, the position and the tilt angle of the antenna were determined to optimise wireless coverage within a $35-\mathrm{m}^{2}$ room. Assumptions are that the receiver (which can be installed in a personal computer above an office desk) and the transmitter are placed with a relative height difference of $1.4 \mathrm{~m}$ and that the receiver antenna is always oriented for maximum reception $(10 \mathrm{dBi})$. Also, because of the relatively high attenuation that occurs at $60 \mathrm{GHz}$, reflection effects were neglected. Simulation results illustrated in Fig. 4 show that the best coverage is obtained when the RF beam is aligned along the longitudinal dimension of the reference rectangular cell with a tilt angle of $30^{\circ}$ (Fig. $4 \mathrm{c}$ ).

A pico-cellular 60-GHz Radio-over-Fibre system demonstrator intended for the domestic market is under development in the aforementioned project ORIGIN. The aim is to demonstrate the feasibility of a multipoint-to-multipoint low-cost bidirectional in-building RoF system with a bit rate of up to $6.7 \mathrm{Gbps}$ and operating at $57-66 \mathrm{GHz}$. In order to demonstrate the functionality of such a system, four rooms in a house used as a laboratory will be equipped with transmit and receive (TRX) radio modules which are then interconnected using an optical or electrical switching network.

Although the system operates in the $60-\mathrm{GHz}$ range, the use of an intermediate frequency of less than $10 \mathrm{GHz}$ allows the project to profit from cheaper and commercially available components for the optical part of the system. Generally, low-cost lasers and photodiodes can handle a bit rate of up to $10 \mathrm{Gbps}$ which defines the intermediate frequency. The fibre chosen is a low-cost multi-mode fibre which is easier and cheaper to install than a single-mode fibre. The $60-\mathrm{GHz}$ receive and transmit modules are designed within the project using commercially available $60-\mathrm{GHz}$ monolithic microwave integrated circuits (MMIC) from Goteborg Microwave Integrated Circuits and Hittite Microwave Corporation. The mm-wave signal of these modules is converted to/from the intermediate frequency by a mixer in the MMIC. The system architecture for the demonstrator using a first point-to-point setup is presented in Fig. 5. The architecture in Fig. 5 is bidirectional and an electrical or optical $4 \times 4$ switch module is used to dispatch the signal in every direction to establish the multipoint-to-multipoint system.

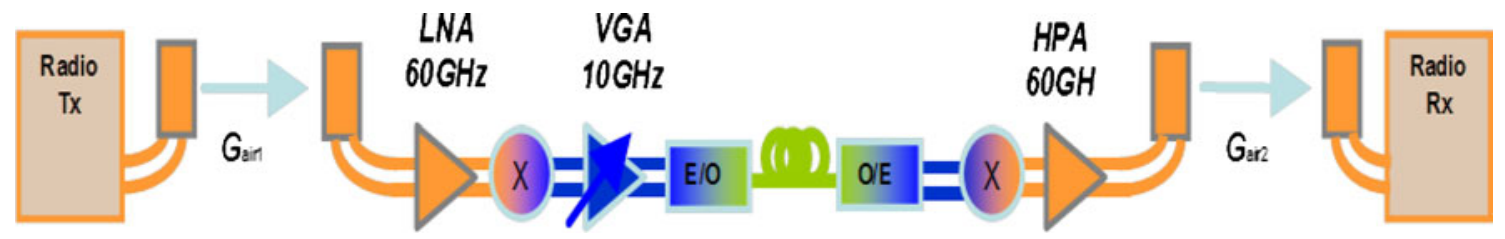

Fig. 5 The system architecture of the ORIGIN demonstrator. Point-to-point variant. Orange parts work at $60 \mathrm{GHz}$, blue parts at the intermediate frequency and green parts are optical components 


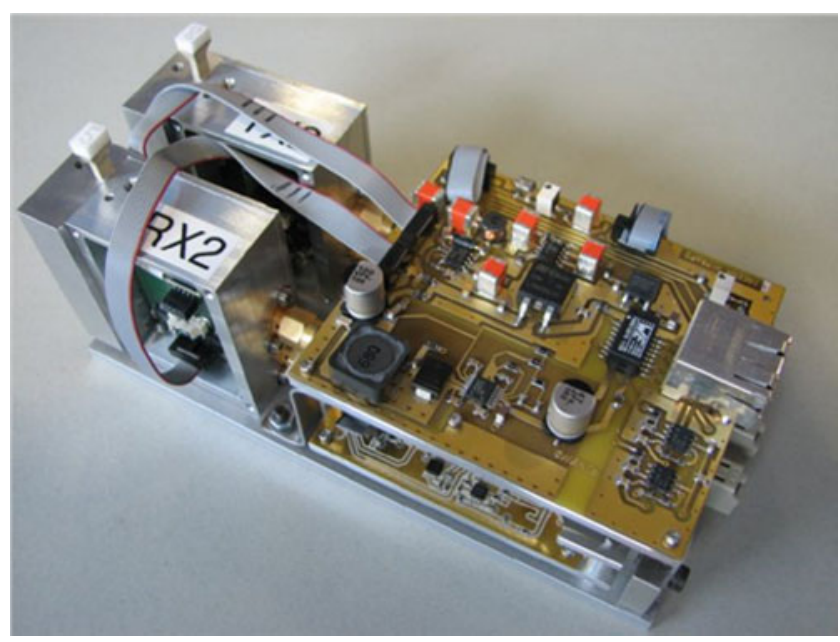

Fig. 6 Second version of the ORIGIN system demonstrator module

In spring 2011, a first demonstration of this system was accomplished by using first-generation modules of $\mathrm{cm}$ wave $(5 \mathrm{GHz})$ and $\mathrm{mm}$-wave $(60 \mathrm{GHz})$ radio modules while using commercially available opto-electrical components (laser and photodiode). In February 2012, a second system demonstration was successfully achieved using the second generation of cm- and mm-wave modules and, for the first time, project-specific developed opto-electrical components. This project is ongoing, and improvements are made as the system components are replaced by next generation components. Figure 6 shows the second generation of the ORIGIN transceiver module. A full description of the architecture and measurement data for different test cases can be found in [42-46]. In [45], a real-time transmission between two commercial Wireless HD devices at $3 \mathrm{Gbps}$ is achieved with a separation distance of up to $5 \mathrm{~m}$ between the access points and the 60-GHz terminals. The fibre used (OM3 multi-mode fibre) can be up to $300 \mathrm{~m}$ long, which is far more than one probably ever needs in a domestic case.

\subsection{Discussion}

It is clear that distributed antenna systems can advantageously provide extended wireless coverage with lower transmit power levels and increased date rates, as compared to a conventional single antenna system. From the sustainability viewpoint, however, the increased number of wireless access points might not be reasonable to cut down on greenhouse gas emissions due to the production, operation and recycling of such an increased number of devices. A consistent approach to attain the energy efficiency of the transmit power and all the network components is required.

For illustrative purposes, the simple model described in the preceding section is applied to a large room $(10 \times$ $8=80 \mathrm{~m}^{2}$ ) within the SITE building of the University of
Ottawa. The transmit powers of a DAS and a single antenna system at $60 \mathrm{GHz}$ operating at data rates according to the IEEE 802.15.3c standard [47] are compared. In this case, the path loss and shadowing term add a total of over $80 \mathrm{~dB}$ in losses at the carrier frequency under study. As illustrated in Fig. 7a, a single antenna system with a transmit power of $13 \mathrm{dBm}$ can only cover around $35 \%$ of the room area for a received power of at least $-70 \mathrm{dBm}$ (which corresponds to a data rate of at least $32.1 \mathrm{Mbps}$ according to the IEEE 802.15.3c standard). In comparison, a DAS system comprised of eight antennas uniformly positioned across the room, as shown in Fig. $7 \mathrm{~b}$ and with $0 \mathrm{dBm}$ of transmit power per antenna, can cover over $98 \%$ of the area at the same minimum data rate and $95 \%$ at $1.54 \mathrm{Gbps}$. In addition, the coverage for a higher received power of $-50 \mathrm{dBm}$ (which corresponds to a data rate of 5,005 Mbps according to the IEEE Standard 802.15.3c) is increased from $10 \%$ for the single antenna to $48 \%$ in a DAS.

Taking into account the same area of coverage, a rough comparison can also be established between the $2.4 \mathrm{GHz}$
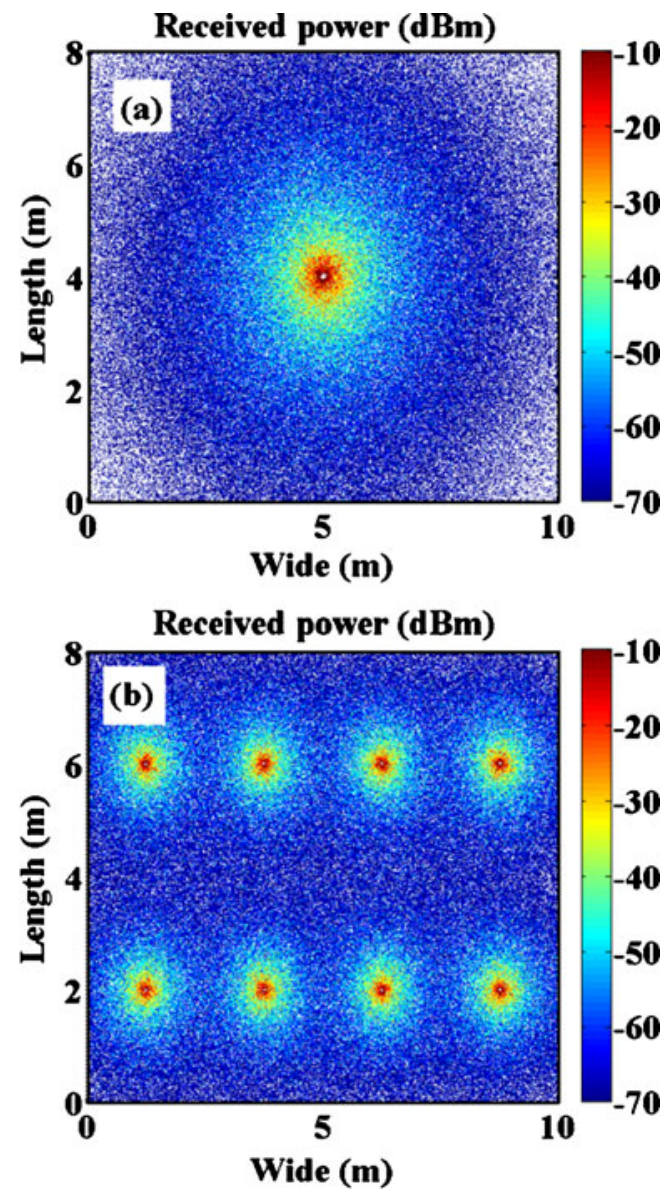

Fig. 7 Received power (in decibel-milliwatt) by a mm-wave terminal operating under the IEEE Standard 802.15.3c, considering a single access point with a total radiated power of $13 \mathrm{dBm}$ (a) and an eight-antenna system with a total radiated power of $9 \mathrm{dBm}(\mathbf{b})$ 
and the mm-wave systems. Whilst the former system can provide a data rate of $54 \mathrm{Mbps}$ utilising only one antenna with $5 \mathrm{dBm}$ of transmit power for a coverage of $\sim 110 \mathrm{~m}^{2}$ (refer to the coverage illustrated by the red circles (54 Mbps) in Fig. 2b), a distributed scheme of eight antennas (1 $\mathrm{mW}$ of transmit power per antenna) would be necessary for a mmwave system covering $\sim 100 \mathrm{~m}^{2}$ (see Fig. $7 \mathrm{~b}$ ), albeit the data rate is significantly higher (1.54 Gbps).

This analysis has been extended to the whole SITE building. The current 2.4-GHz wireless network runs with around 100 access points (IEEE $802.11 \mathrm{~g}$ ) with $15 \mathrm{~mW}$ of transmit power per node and data rate of $54 \mathrm{Mbps}$. It is estimated that the same coverage can be reached, installing around 1,000 access points at $60 \mathrm{GHz}$ (IEEE 802.15.3c) with $1 \mathrm{~mW}$ per node and data rate of $1.54 \mathrm{Gbps}$. Under these conditions, a distributed antenna system at $60 \mathrm{GHz}$ will consume $33 \%$ less power relative to the existing $802.11 \mathrm{~g}$ network and provide a service with a data rate of $1.54 \mathrm{Gbps}$. The reduction in energy consumption is justified by the fact that power dissipation is proportional to the RF transmitted power. Hardware and auxiliary equipment may be characterised by a power efficiency, which for a mobile base station is as low as $10 \%$. A more accurate model might result in a constant plus a linear part, even that constant is likely to be proportional to the designed capacity of the transmitter. In the distributed case, the fact that each transmitter may operate at reduced power means that the remainder of the hardware should be designed to operate at a commensurately reduced power, attempting to preserve equipment efficiency.

On the other hand, further energy savings may be reached through dynamic allocation of resources (power and data on demand) in time and space, for instance, by ensuring that distributed antenna units are only fully powered on when and where resources are needed, which is essentially a crude form of directionality (connect to nearest antenna only).

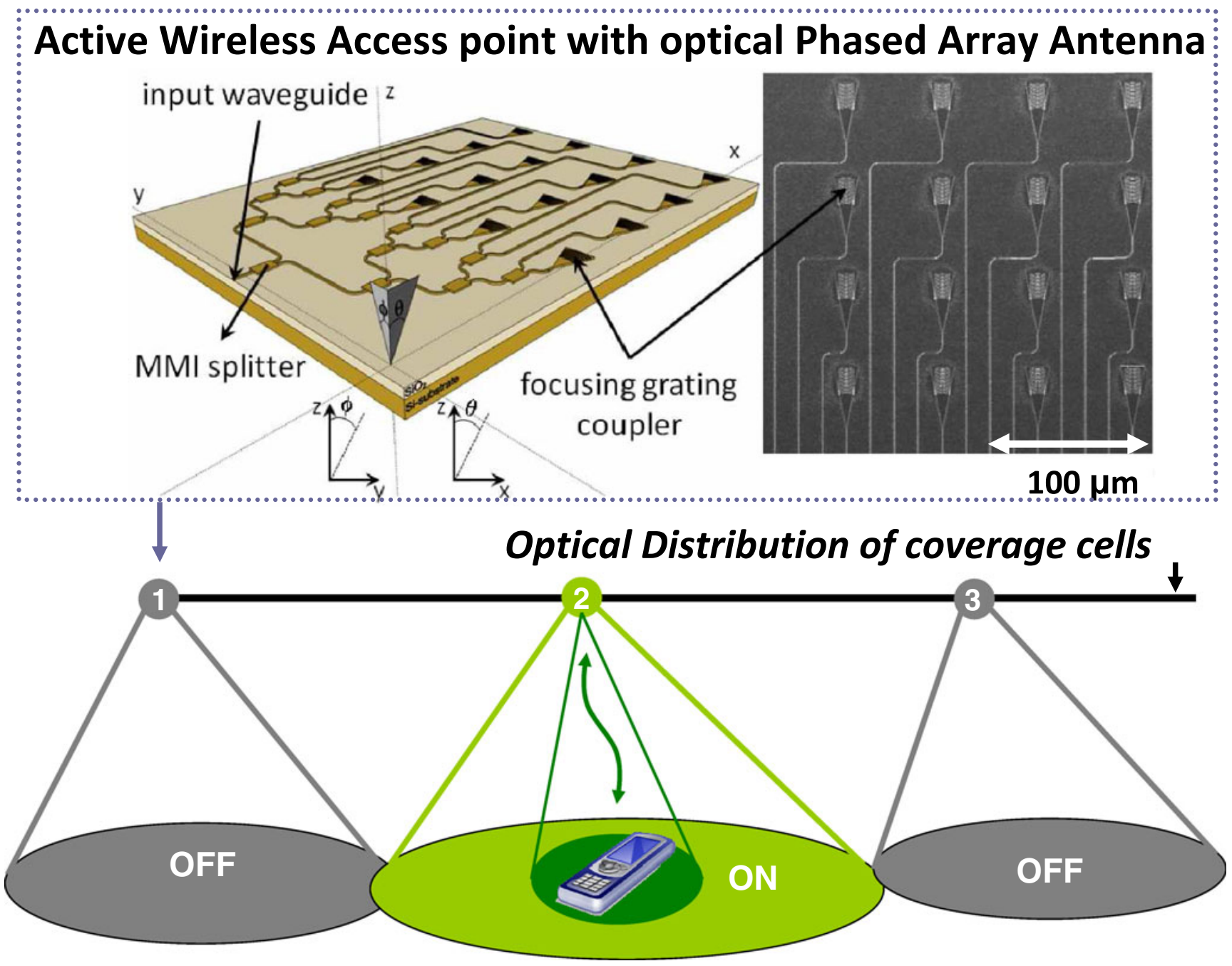

Fig. 8 Fibre-distributed antenna system with phased array antennas. Inset after [48] 
To attain further reduction in energy consumption, small antenna arrays (i.e. with dimensions of the order of 200300 micrometres), as illustrated in Fig. 8, can be advantageously used to form and steer beams as in phased array radars. Ideally, both transmitter and receiver antennas would use phased array techniques to set up highly directional and thus energy-efficient point-to-point links. This creates a formidable multiple target acquisition and tracking problem.

Deploying arrays with a significant number of elements is neither economical nor practical (especially at the mobile terminal) for nonmilitary use at micro- or $\mathrm{mm}$-wave frequencies (for optical frequencies see [48]). Nevertheless, the multiple-input multiple-output (MIMO) technique can be considered to combine diversity reception for robustness against fading signals and adaptive beam forming for enhanced channel capacity [49]. MIMO clearly contributes to improved energy efficiency and is being deployed widely.

\section{Digital coherent optical Radio-over-Fibre}

The distributed antenna system requires a feeder network. It is well established that optical fibre networks offer orders of magnitude lower energy consumption compared to other technologies. Considerations of reduced energy consumption of radio access networks in a Digital City have led us inexorably to the Radio-over-Fibre paradigm [50].

RoF has long been viewed as a means to simplify remote antenna units, base stations and the backhaul network that connect to the central office. RF transport over analogue intensity modulation direct detection (IM-DD) optical links has dominated RoF research so long that 'analogue transport' and 'RoF' have become synonymous, but analogue transport is subject to problems of noise, linearity, dynamic range and dispersion. The industry preference is for digital RF transport. The arguments herein support a distributed antennas system driven by a fibre optical network but are silent on the best mode of operation.

To accommodate bandwidth-hungry applications, wireline systems must devote an entire wavelength to the signal as in wavelength division multiplexed (WDM) passive optical networks (PON). One consequently envisages a converged Radio-over-Fibre WDM-PON paradigm in which RF signals are distributed (in analogue or digital form) to wireless access points over an optical fibre network that also offers wired access. The optical fibre may also be used to distribute low-noise optical carriers for use as local oscillators at the optical access points or to provide mmwave carriers at the wireless access points. Owing to the higher path loss at mm-wave frequencies, transmission has a limited coverage (e.g. confined within a room), which, on the other hand, provides the advantage of frequency reuse and the conception of smaller antennas (140 times smaller) relative to their commercial counterpart currently in operation.

\subsection{Analogue vs. digital transmission}

The most effective RoF operating mode remains an open question. It is commonly assumed that RoF employs analogue IM-DD optical links. However, all methods of intensity modulation have inherent nonlinear transfer functions and hence are subject to problems of noise, linearity, dynamic range, and dispersion. One option is to operate with a small modulation index but that exacerbates the signal-to-noise ratio and energy efficiency issues. Another option is to compensate the nonlinearity of the link through a combination of pre-distortion and/or post-distortion processing, and to exploit symmetries to suppress third-order distortion arising from the modulator [51].

RF base stations already contain much digital processing in the radio equipment control (REC). One, therefore, has the option of using digital signal processing (DSP) to correct for analogue impairments. Linearization by DSP shows considerable promise [52] to permit operation in regimes for which the RF amplifier is efficient but normally distorts excessively.

Another aspect deals with the transport of analogue broadband RF signals. Although this scheme offers easy transparency to microwave signal formats, the requirement to terminate a high-speed photodiode directly into a load matched to the low characteristic impedance of a waveguide $[53,54]$, results in a low electro-optic conversion efficiency and poor noise figure mandating quite high transmitted optical power $(\sim 0 \mathrm{dBm})$. In addition, the differential delays introduced by the chromatic dispersion of the fibre can also be a cause of impairments to broadband RF modulation by changing the relative phase relationship between frequency components (e.g. carrier and sidebands). In the case of remote heterodyning architectures, for instance, the differential delays may also cause the de-correlation of the phase noise of the two optical signals that beat to create the local oscillator, resulting in a noisier and weaker beat [55]. Therefore, the principal challenge has been to achieve high linearity and hence a high spurious-free dynamic range.

\subsection{Digitised Radio-over-Fibre}

RF signals use advanced modulation formats that are synthesised in DSP hardware (e.g. orthogonal frequency division multiplexing uses a fast Fourier transform), and the analogue RF is reconstructed using a digital-to-analogue converter (DAC). There is consequently a digital interface between the REC and radio equipment (RE) which has motivated a digitised RF-over-fibre (D-RoF) approach 
[56]. The analogue RF input signal is first digitised by an analogue-to-digital converter (ADC), then transported over a binary shift keyed IM-DD optical link, and finally reconstructed by a DAC at the output. The D-RoF would lead to a downlink featuring a DAC and ADC connected backto-back with digital RF-in and digital RF-out in different formats. This ensures that the optical link is transparent to the RF protocol; functionally an identity mapping, the data formats differ at input and output of the DAC-ADC but the encoded RF signal is unaltered. Despite the complexity, there are indications that digitisation offers lower energy consumption primarily because a more sensitive photoreceiver may be used [57].

\subsection{Digital coherent optical transmission}

An alternative approach retains analogue transport, but phase modulation is used to exploit the inherent linearity of phase modulators that make use of the electro-optic effect $[58,59]$. Phase modulation necessarily requires the use of coherent detection. Once the coherent detection in $\mathrm{RoF}$ is embraced, it is a small step to the use of modern digitally enabled coherent optical transmission [60]. Optically transporting the wireless protocol natively provides some simplification. That it should work is not surprising given that the advanced modulation formats gaining popularity in optical communications were first used in wireless. Essentially, the downlink transports the RF signal on a carrier at optical frequencies and this is down-converted (by the optical local oscillator, LO) to an RF carrier at the remote antenna unit, where it might be further translated in frequency using an RF LO.

Coherent transmission can increase the capacity, and the unamplified reach of the optical link (the receiver requires $-20 \mathrm{dBm}$ rather than $1-10 \mathrm{dBm}$ of optical power), hence a net power consumption/unit bandwidth saving, especially through the elimination of power-hungry Erbium-doped fibre amplifiers or RoF repeaters. Moreover, it is also possible to compensate the frequency drift of an optical local oscillator (the linewidth requirements are also eased somewhat) and the transmission impairments (e.g. chromatic dispersion, nonlinearity) entirely in the digital domain [61, 62]. Prima facie, this approach is well suited to the uplink. Wireless protocols have, however, time-out mechanisms that militate against long-reach architectures [63].

The cost of the coherent systems discussed is falling rapidly with advances in photonic integration of the coherent optical front-end and mixed signal integration in CMOS VLSI. Widespread adoption of these techniques will occur if the DSP blocks are implemented in ASICS technologies. This would also dramatically reduce the energy consumption of the transmission system by an order of magnitude or more.

Different solutions for upstream and downstream may offer a greater simplification of the antenna units. The implementation of polarisation diverse schemes is an essential consideration. Access to the field yields a linear channel that is a composition of the wireless and optical channels which facilitates impairment correction by digital signal processing. A local oscillator may be distributed optically using the same feeder network as the signal. Information may be carried in the amplitude, phase and polarisation of the optical field. Additionally, the selectivity of a coherent receiver is very well suited to dense multiple access networks.

\subsection{Numerical simulations of a digital coherent RoF system}

To illustrate the importance of exploiting modern digital coherent optical transmitters and receivers in multi-Gbps RoF systems relative to the traditional intensity modulation direct detection schemes, a numerical simulation of a coherent RoF system carrying 10-Gbps QPSK signals has been implemented. The adopted architecture for the downlink and the uplink is illustrated in Fig. 9. The simulation software used for this purpose is VPI Transmission Maker $^{\mathrm{TM}}$.
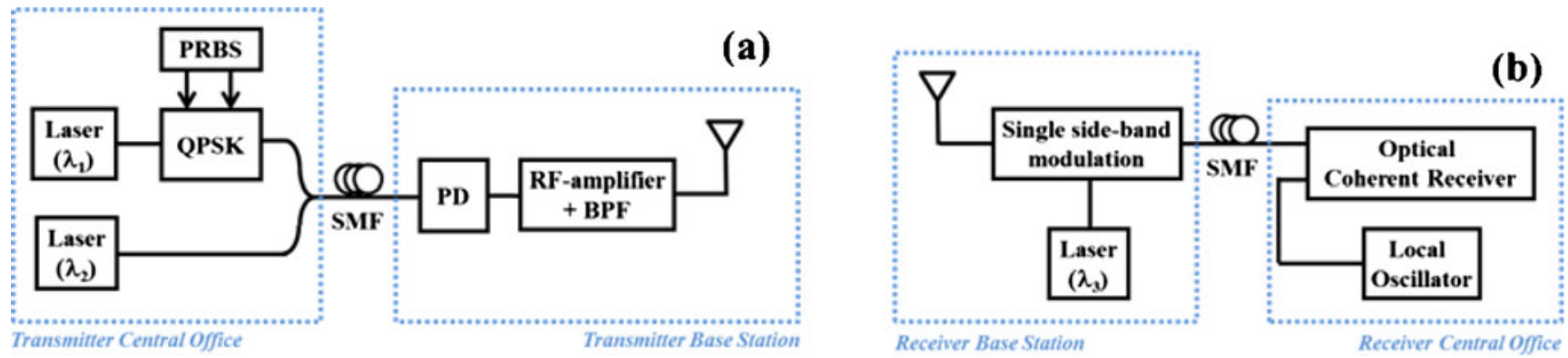

Fig. 9 Adopted architecture in the numerical simulation. Downlink (a) and uplink (b). $P R B S$, pseudo-random binary sequence; $Q P S K$, quadrature phase shift keying; $S M F$, single mode fibre; $P D$, photodetector; $R F$, radio frequency; $B P F$, band-pass filter 
Table 2 Summary of component specifications utilised in the numerical simulations: downlink

\begin{tabular}{ll}
\hline Externally modulated laser & \\
Wavelength & $\lambda_{1}=1550 \mathrm{~nm}$ \\
FWHM linewidth & $\Delta \mathrm{f}=1 \mathrm{MHz}$ \\
Average power & $P_{1}=20 \mathrm{~mW}$ \\
Continuous wave laser & \\
Wavelength & $\lambda_{2}=1549.52 \mathrm{~nm}$ \\
FWHM linewidth & $\Delta \mathrm{f}=1 \mathrm{MHz}$ \\
Average power & $P_{2}=5 \mathrm{~mW}$ \\
Photodiode & \\
Responsivity & $\mathrm{R}=0.65 \mathrm{~A} / \mathrm{W}$ \\
Frequency bandwidth & $\mathrm{B}=110 \mathrm{GHz}$ \\
RF amplifier & \\
Linear gain & $\mathrm{G}=20 \mathrm{~dB}$ \\
Frequency bandwidth & $\mathrm{B}=110 \mathrm{GHz}$ \\
Electrical band-pass filter & \\
Centre frequency & $\mathrm{f}=60 \mathrm{GHz}$ \\
Frequency bandwidth & $\mathrm{B}=50 \mathrm{GHz}$ \\
\hline
\end{tabular}

\subsubsection{System architecture}

In the downlink (optical to electrical channel, Fig. 9a), a digital baseband signal in a format of a pseudo-random binary sequence (PRBS) externally modulates the phase of a continuous wave $(\mathrm{CW})$ laser at a first wavelength $\lambda_{1}$. A quadrature phase shift-keyed (QPSK) modulation scheme is implemented. After the information channel at $\lambda_{1}$ is combined with a CW laser output at a second wavelength $\lambda_{2}$, both signals $\lambda_{1}$ and $\lambda_{2}$ are transported from the transmitter central office (CO) to the base station (BS) through a span of singlemode optical fibre. A mm-wave carrier is generated at the BS by the heterodyne beating of the two lasers $\left(\lambda_{1}\right.$ and $\left.\lambda_{2}\right)$ into a photoreceiver with a sufficient frequency bandwidth.

Table 3 Summary of component specifications utilised in the numerical simulations: uplink

\begin{tabular}{ll}
\hline Continuous wave laser & \\
Wavelength & $\lambda_{3}=1555 \mathrm{~nm}$ \\
FWHM linewidth & $\Delta \mathrm{f}=1 \mathrm{MHz}$ \\
Average power & $P_{3}=10 \mathrm{~mW}$ \\
Optical band-pass filter for SSB & \\
Centre wavelength & $\lambda_{I F}=1555.48 \mathrm{~nm}$ \\
Bandwidth & $B_{I F}=50 \mathrm{GHz}$ \\
LO at optical coherent receiver & \\
Wavelength & $\lambda_{L O}=1555.48 \mathrm{~nm}$ \\
FWHM linewidth & $\Delta \mathrm{f}_{L O}=1 \mathrm{MHz}$ \\
Average power & $\mathrm{P}_{L O}=3 \mathrm{~mW}$ \\
\hline
\end{tabular}

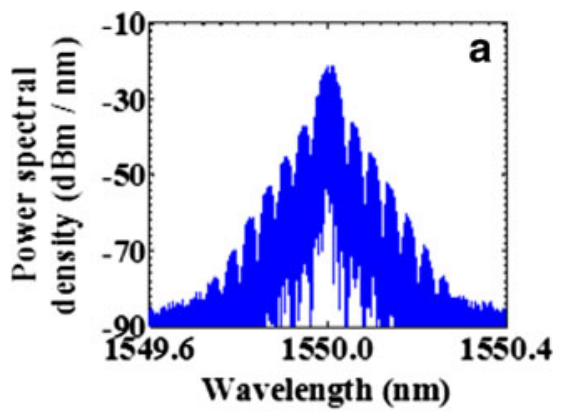

Received power $=-25 \mathrm{dBm}$

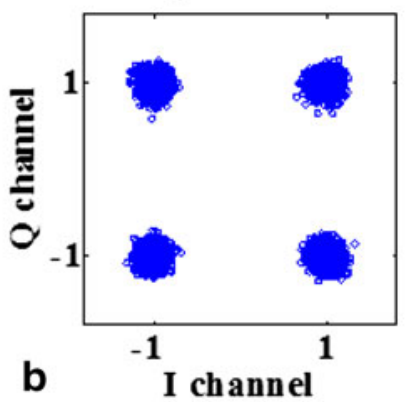

Received power $=-\mathbf{3 0} \mathrm{dBm}$

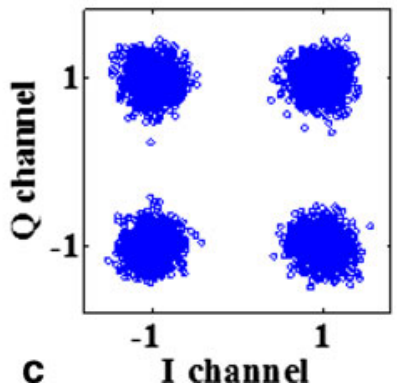

Fig. 10 Optical spectrum (a) and constellation diagrams (b)-(c) of the 10-Gbps QPSK signal transmitted at the downlink

The frequency of the carrier (which is an electrical entity but generated by a photonic approach) is determined by the relative difference between the wavelengths $\left(\lambda_{1}, \lambda_{2}\right)$ of the two lasers and is set to $60 \mathrm{GHz}$ in the present simulations. At the output of the photoreceiver (combination of photodiode, RF amplifier and band-pass filter), the PRBS information channel is hence imprinted on the $60-\mathrm{GHz}$ carrier and can be sent to the end user via a wireless link (not implemented in the simulations but emulated by a given amount of loss).

In the uplink (electrical to optical channel, Fig. 9b), the information channel is converted back to the optical domain after a single sideband modulation scheme. In this case, the intensity of a CW laser at a third wavelength $\lambda_{3}$ is externally modulated by the intermediate frequency (IF) signal at $60 \mathrm{GHz}$. After an optical filtering stage at $\lambda_{3}+\lambda_{I F}$, the resulting signal is transported from the $\mathrm{BS}$ to the $\mathrm{CO}$ and analysed through an optical coherent receiver. 

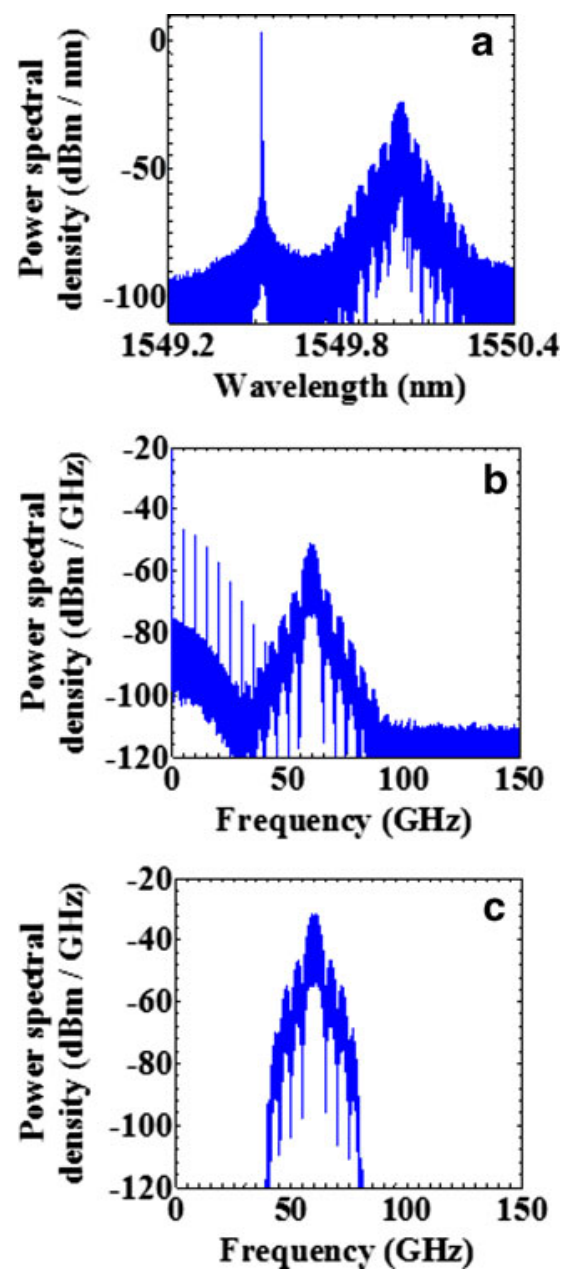

Fig. 11 Spectrum of the combined optical signal at the input of the photodiode (a) spectrum of the signal at the output of the photodiode (b) and after a filtering stage (c)

The specifications of the components utilised in the numerical simulations are set according to the characteristics of actual devices commercially available and summarised in Tables 2 and 3. In particular, semiconductor laser sources with a moderate quality linewidth of $1 \mathrm{MHz}$ are considered. In addition, the use of hungry energy consumption elements such as Erbium-doped fibre amplifiers has been avoided.

\subsubsection{Simulation results}

Preliminary results obtained from the numerical simulations are presented here. The optical spectrum of the 10-Gbps QPSK signal transmitted at the downlink is illustrated in Fig. 10a. In addition, constellation diagrams of such a signal are illustrated in Fig. 10b, c for received powers of -25 and $-30 \mathrm{dBm}$, respectively. In this case, an optical coherent receiver is utilised for obtaining the constellations, and
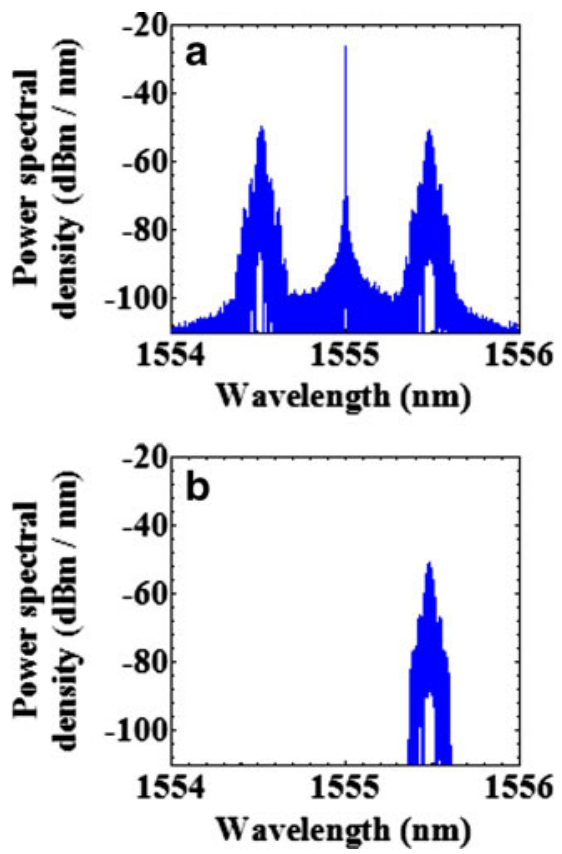

Fig. 12 Optical signal before (a) and after (b) a single sideband filtering stage is implemented to obtain a SSB modulation scheme with carrier suppression

an optical attenuator is included to adjust the power at the input of the detection system.

The QPSK signal depicted in Fig. 10a features an average optical power of $+6.13 \mathrm{dBm}$ and is combined with a continuous wave laser of comparable power by using a $2 \times 1$ optical coupler. The output of the coupler is shown in
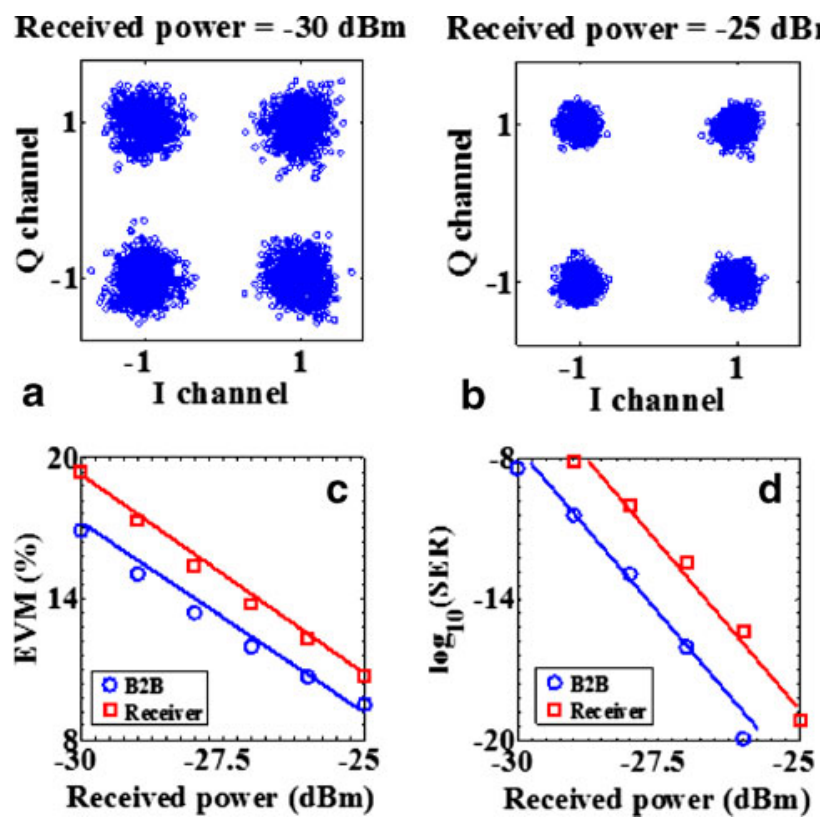

Fig. 13 Constellation diagrams (a-b) and RoF system performance illustrated through the corresponding error vector magnitude (c) and symbol error ratio $(\mathbf{d})$ plots 
Fig. 11a. After their propagation through a span of SMF, a total optical power of $+6 \mathrm{dBm}$ impinges upon a high-speed photodetector. The photocurrent thus generated produces an electrical power of $-1.5 \mathrm{dBm}$ into a $50-\Omega$ load connected at its output. The electrical spectrum obtained at the output of the photodiode is illustrated in Fig. $11 \mathrm{~b}$ and, after a filtering stage, in Fig. 11c. Therefore, the original 10 Gbps PRBS signal is converted from the optical to the electrical domain at an intermediate frequency of $60 \mathrm{GHz}$, which, in this case, is determined by the frequency difference between the two lasers utilised at the downlink. Note that the photonic approach described here for the downlink is advantageous compared to electronic schemes because a $60-\mathrm{GHz}$ electronic oscillator is not required, which greatly simplifies the base station architecture and enables greater flexibility to change carrier frequency. In addition, obtaining an electrical power close to $1 \mathrm{~mW}$ at the output of the photodetector can also be advantageous to avoid the use of electrical amplifiers at the transmitter of the wireless link.

To convert the millimetre-wave carrier to the optical domain, a single sideband (SSB) modulation scheme is implemented in the uplink. For the sake of simplicity and without loss of generality, a double sideband modulation followed by a filtering of one sideband is implemented in the present numerical simulation. However, a significant improvement and further research on efficient SSB approaches is anticipated. The optical signal before and after the single sideband filtering stage is illustrated in Fig. 12.

After the optical SSB modulation is implemented, the signal is detected and analysed by an optical coherent receiver. Constellation diagrams of the received signals are illustrated in Fig. 13a, b for received powers of -30 and $-25 \mathrm{dBm}$, respectively. In addition, the corresponding error vector magnitude and symbol error ratio are shown in Fig. 13c, d, respectively.

Despite the fact that numerical simulation results shown in Fig. 13 are obtained without elaborated digital signal processing and therefore are subject to further improvement, the symbol error ratio of the received signal indicates a power penalty of $+1 \mathrm{~dB}$ relative to its back-to-back value. Such a result demonstrates the feasibility for applying optical coherent techniques in Radio-over-Fibre systems demanding transmission rates of several Gbps in addition to a system operation with a reduced consumption of energy.

Last but not the least, the numerical results presented here are supported by experimental investigations currently

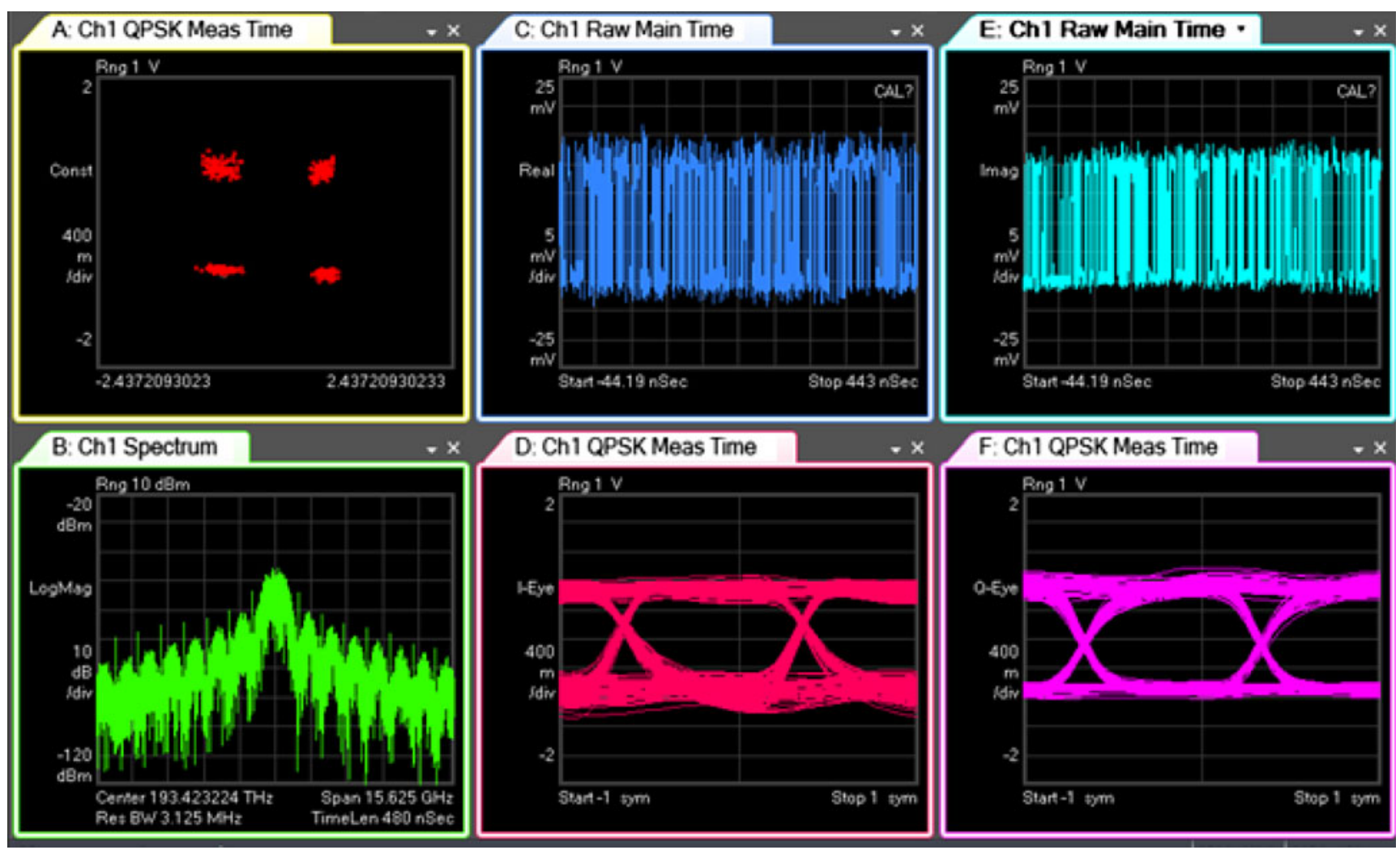

Fig. 14 Optical spectrum and constellation diagram of a QPSK optical signal at 5 Gbaud recorded by an optical coherent receiver for an input power of $-5 \mathrm{dBm}$. Time domain traces and eye diagrams of the in-phase and quadrature channels are also illustrated 
in progress in the Photonic Technology Laboratory of the Center for Research in Photonics at the University of Ottawa. A sample of an actual constellation diagram of a 5-Gbaud PRBS optical signal is illustrated in Fig. 14 (top left trace), which is recorded by an optical coherent receiver for an input power of $-5 \mathrm{dBm}$. Separate time domain traces for the in-phase and in-quadrature channels in addition to their corresponding eye diagrams are also shown in Fig. 14.

\subsubsection{Discussion}

The proceeding architecture relied on the heterodyne beating of two independent laser sources on a high-speed photodetector. The efficacy of this approach depends upon the stability, linewidth and degree of phase correlation between the lasers. Frequency drift of the lasers directly translates to frequency drift of the carriers. A broad linewidth and low degree of correlation can result in an unacceptably noisy carrier signal.

An ideal mm-wave source would be frequency agile, tuneable and incorporated directly into a system to replace multiple banks of conventional signal generators. Indeed, the availability of inexpensive integrated $\mathrm{mm}$-wave sources is an enabling factor for the deployment of wireless RoF communications systems operating at frequencies around $30 \mathrm{GHz}$ and up to $110 \mathrm{GHz}$. One approach to photonic means of achieving this ideal is to use heterodyne beating of a master and a slave lasers. Phase-locked loop techniques may then be used to control the frequency of the slave laser, so that the beat is locked to a harmonic of a micro- $/ \mathrm{mm}$ wave reference [64]. Alternatively, narrow linewidth single frequency lasers may be phase-locked by optical injection locking to individual lines separated by the desired RF frequency of a frequency comb generated by mode-locking a semiconductor laser. Quantum dot lasers show particular promise in this respect. If necessary, the spacing of the lines of the comb may be locked to a lower-frequency microwave reference source. Much of the complexity of these methods vanishes if a laser can be made that oscillates stably in two longitudinal modes separated in frequency by the desired RF frequency. The hope is that the two modes share the same electrical, thermal and mechanical fluctuations. The linewidth of the beat between the two modes will be then significantly narrower than the linewidth of the individual modes because of the correlation of their phase noise [65]. Simultaneous emission from two modes within the same laser cavity is very appealing due to its compactness, stability and spectral quality. However, the two modes will also compete with each other for a share of the gain, and this can lead to the instability of two-mode operation.

\section{Conclusions}

Energy efficiency in telecommunication networks (in general) and in broadband wireless access networks (in particular) must be carefully analysed by engineers and industry partners, as well as jointly discussed with urban planners for the conception of sustainable Digital Cities.

Based on the fact that the total power consumption of a base station varies proportionally to the radiated RF power, the distributed antenna systems are likely the only solution for reducing energy consumption in cluttered access networks providing broadband links at millimetre-wave carriers. From the sustainability viewpoint, however, the increased number of wireless access points might not be effective to cut down on greenhouse gas emissions due to the production, operation and recycling of such an increased number of devices. Therefore, a consistent approach to attain the energy efficiency of the transmit power and all the network components is required. On the other hand, the fibre optic feed access networks hence conceived require the deployment of advanced wireless protocols to cope with time-out mechanisms that militate against long-reach architectures.

Energy-efficient distributed antenna architectures in broadband wireless access networks are complemented by an optical digital coherent system. The inherent linearity and increased capacity of such a system dictates a radical improvement relative to the traditional analogue intensity modulation direct detection implementation.

The enabling factor of millimetre-wave broadband access architectures points towards solutions in two main aspects: the generation of the millimetre-wave carriers at the remote antenna unit (downlink) and an efficient implementation of single sideband modulation (uplink). While the photonic generation of millimetre-wave carriers seems to be a feasible approach, their ultimate advantages relative to electronic techniques remain an open question in terms of performance and cost. Improved approaches for implementing the single sideband modulation are needed to mitigate the conversion losses and the stringent control of temperature.

Acknowledgments This research was initiated when Dr. Trevor Hall was on sabbatical leave hosted by Télécom Bretagne France between 1st July 2010 and 30 June 2011, when he held the SISCom International Chair supported by the Université Européenne de Bretagne. Dr. Hall is also grateful to the Canada Research Chairs programme for their support.

Open Access This article is distributed under the terms of the Creative Commons Attribution License which permits any use, distribution, and reproduction in any medium, provided the original author(s) and the source are credited. 


\section{References}

1. Kim Y, Jeong BJ, Chung J, Hwang C-S, Ruy JS, Kim K-H, Kim YK (2003) Beyond 3G: vision, requirements, and enabling technologies. IEEE Commun Mag 41:120-124

2. Novak D (2004) Fiber optics in wireless applications. In: Optical fibre communications conference (OFC 2004), short course, p 217

3. Wake D (2002) Radio over fibre systems for mobile applications. In: Al-Raweshidy H, Komaki S (eds) Radio over fiber technologies for mobile communication networks. Artech House, Inc., USA

4. Yao J (2009) Microwave photonics. IEEE J Lightwave Technol 27(3):314-335

5. Seeds AJ, Williams KJ (2006) Microwave photonics. IEEE J Lightwave Technol 24(12):4628-4641

6. Sauer M, Kobyakov A, George J (2007) Radio over fiber for pico cellular network architectures. J Lightwave Technol 25(11):33013320

7. Smart Cities Workshop (2009) EUROCITIES. Brussels, 16-17 Nov

8. Statistics Canada (2012) Population, urban and rural, by province and territory (Ontario). http://www.statcan.gc.ca/tables-tableaux/ sum-som/101/cst01/demo62g-eng.htm. Accessed Oct 2012

9. http://www.urenio.org. Accessed Aug 2012

10. http://www-03.ibm.com/innovation/us/thesmartercity. Accessed Aug 2012

11. http://www.greenbusinesstimes.com/2010/04/27/ siemens-city-of-the-future. Accessed Aug 2012

12. http://www.meaningfulinnovation.philips.com/Livable-Cities. Accessed Aug 2012

13. NiCE Networking Intelligent Cities for Energy Efficiency (20112014). http://www.eurocities.eu/eurocities/activities/projects/ NiCE-Networking-intelligent-Cities-for-Energy-Efficiency. Accessed Aug 2012

14. Pickavet M, Tucker R (2008) Network solutions to reduce the energy footprint of ICT. In: European conference on optical communications (ECOC 2008), Brussels, Belgium, 21-25 Sept 2008

15. http://www.itac.ca/uploads/events/greenict-april27-2010/ CharlesDespinsPresentation.pdf

16. Marsan MA, Chiaraviglio L, Ciullo D, Meo M (2009) Optimal energy savings in cellular access networks. In: IEEE international conference on communications workshops (ICC workshops), 1418 June 2009

17. Cucchietti F, Griffa G, Radice L (2007) Eco-efficiency indicator: an operator's energy performance indicator. In: 29th international telecommunications energy conference (INTELEC), 30 Sept-4 Oct 2007, pp 743-748

18. European Commission Joint Research Center (2010) Major ICT companies join European Commission initiative to reduce electricity consumption, 28 Sept 2010. www.eurekalert.org/pub-releases/ 2010-09/ecjr-mic092810.php. Accessed Aug 2012

19. An Inefficient Truth (2007) Global action plan. http://www. globalactionplan.org.uk/green-it. Accessed Aug 2012

20. Asami T, Namiki S (2008) Energy consumption targets for network systems. In: European conference on optical communications (ECOC 2008), Brussels, Belgium

21. Walker M (2008) Surfing the green wave in telecom. OVUM

22. Cooperson D, Mazur J, Walker M (2009) Increased focus on network power consumption to lower OpEx, Go Green. OVUM

23. Jevon's Paradox Wikipedia. http://en.wikipedia.org/wiki/ Jevons-paradox. Accessed Aug 2012

24. http://www.iisd.org/. Accessed Aug 2012

25. http://www.canarie.ca/. Accessed Aug 2012
26. Foll LS (2008) TIC et Énergétique: Techniques d'estimation de consommation sur la hauteur, la structure et l'évolution de l'impact des TIC en France. Version publique. PhD Thesis, Institut National des Télécommunications (Telecom and Management SudParis)

27. Gladisch A, Lange C, Leppla R (2008) Power efficiency of optical vs electronic access networks.. In: European conference on optical communications (ECOC 2008), Brussels, Belgium, 21-25 Sept 2008

28. Alping A (2010). Researcher, Principal, Broadband Interconnects at Ericsson AB, Sweden. Private Communication

29. www.greentouch.org

30. EARTH 'Energy-aware radio and neTwork technologies' (20102012) Improving the energy efficiency of wireless networks through cognitive and reconfigurable radio technology. www. ict-earth.eu

31. OPERA-Net I (2008-2011) Optimising power efficiency in mobile radio networks. http://opera-net.org

32. OPERA Net II (2011-2014) Optimising power efficiency in mobile radio networks 2. www.celtic-initiative.org/Projects/ Celtic-Plus-Projects/2011/OPERA-NET2/operanet2-default.asp

33. European Network of Excellence TREND Towards real energyefficient network design. www.fp7-trend.eu (see WP2 on energy efficient access networks)

34. Etoh M, Ohya T, Nakayama Y (2008) Energy consumption issues on mobile network systems. In: International symposium on applications and the internet, (SAINT), 28 July-1 Aug 2008, pp 365-368

35. Chow P, Karim A, Fung V, Dietrich C (1994) Performance advantages of distributed antennas in indoor wireless communication systems. In: IEEE 44th vehicular technology conference, vol 3, pp 1522-1526

36. Richter F, Fehske AJ, Fettweis GP (2009) Energy efficiency aspects of base station deployment strategies for cellular networks. In: IEEE 70th vehicular technology conference fall (VTC 2009Fall), 20-23 Sept 2009, pp 1-5

37. Yong S-K, Xia P, Garcia AV (2011) Chapter 1: introduction to $60 \mathrm{GHz}$ and chapter 2: $60 \mathrm{GHz}$ channel characterizations and modeling. In: $60 \mathrm{GHz}$ technology for Gbps WLAN and WPAN: from theory to practice. Wiley, Chichester

38. Ebert J, Burns B, Wolisz A (2002) A trace-based approach for determining the energy consumption of a WLAN network interface. In: Proc. of European wireless, Florence, Italy

39. Josse Y, Fracasso B, Lucarz F, Pajusco P (2011) Model for energy efficiency in radio over fiber distributed indoor antenna Wi-Fi network. In: WPMC'11: 14th international symposium on wireless personal multimedia communications, Brest, France, 3-7 Oct 2011

40. Weiss M, Stohr A, Huchard M, Fedderwitz S, Charbonnier B, Rymanov V, Babiel S, Jager D (2008) 60 GHz Radio-over-Fibre wireless system for bridging $10 \mathrm{~Gb} / \mathrm{s}$ ethernet links.. In: European conference on optical communication (ECOC 2008), 21-25 Sept 2008, pp 1-2

41. Bikiny A, Bourreau D, Karnfelt C, Ney M (2011) Antenne cornet en technologie mousse pour une application radio sur fibre a 60 GHz. In: JNM 2011: Journées Nationales Micro-ondes, Brest, France, 18-20 May 2011

42. Guillory J, Meyer S, Sianud I, Ulmer-moll AM, Charbonnier B, Pizzinat A, Algani C (2010) Radio-over-Fibre architectures. IEEE Veh Technol Mag 5(3):3038

43. Guillory J, Tanguy E, Pizzinat A, Charbonnier B, Meyer S, Algani C, Li H (2011) A $60 \mathrm{GHz}$ wireless home area network with radio over fibre repeaters. IEEE J Lightw Technol 29(16):2482-2488

44. Guillory J, Tanguy E, Pizzinat A, Charbonnier B, Meyer S, Li HW, Algani C (2011) Radio over fibre tunnel for $60 \mathrm{GHz}$ wireless home network. In: Optical fibre communication conference (OFC 2011) 
45. Guillory J, Pizzinat A, Charbonnier B, Algani C (2011) $60 \mathrm{GHz}$ intermediate frequency over fibre using a passive multipoint-tomultipoint architecture. In: 16th European conference on networks and optical communications (NOC), pp 44-47

46. Guillory J, Richard F, Guignard P, Pizzinat A, Meyer S, Charbonnier B, Guillo L, Algani C, Li HW, Tanguy E (2011) Towards a multiservice and multiformat optical home area network. In: 14th ITG conference on electronic media technology (CEMT), pp 1-6

47. IEEE Standard for Information Technology-Telecommunications and information exchange between systems-Local and metropolitan area networks-Specific requirements. Part 15.3: Wireless Medium Access Control (MAC) and Physical Layer (PHY) Specifications for High Rate Wireless Personal Area Networks (WPANs), Amendment 2: Millimetre wave-based Alternative Physical Layer Extension, IEEE Standard 802.15.3c-2009 (Amendment to IEEE Std 802.15.3-2003), pp. C1-187, Oct. 12, 2009

48. Van Acoleyen K, Rogier H, Baets R (2010) Two-dimensional optical phased array antenna on silicon-on-insulator. Opt Express 18(13):13655-13660

49. Gesbert D, Shafi M, Shiu D-S, Smith PJ, Naguib A (2003) From theory to practice: an overview of MIMO space-time coded wireless systems. IEEE J Sel Areas Commun 21(3):281-302

50. Lim C, Nirmalathas A, Bakaul M, Gamage P, Lee K-L, Yang Y, Novak D, Waterhouse R (2010) Fiber-wireless networks and subsystem technologies. J Lightw Technol 28(4):390-404

51. Li S, Zheng X (2010) Highly linear radio-over-riber system incorporating a single-drive dual-parallel Mach-Zehnder modulator. IEEE Photonics Technol Lett 22(24):1775-1777

52. Hekkala A, Lasanen M, Harjula I, Vieira LC, Gomes NJ, Nkansah A, Bittner S, Diehm F, Kotzsch V (2010) Analysis of and compensation for non-ideal RoF links in DAS [coordinated and distributed MIMO]. IEEE Wirel Commun 17(3):52-59

53. Fu Y, Pan H, Campbell JC (2010) Photodiodes with monolithically integrated Wilkinson power combiner. IEEE J Quantum Electron 46(4):541-545

54. Bach H-G (2007) Ultrafast efficient photodiodes exceeding $100 \mathrm{GHz}$ bandwidth (Invited). In: 2007 international conference on indium phosphide and related materials, Matsue, Japan, 14-18 May 2007, pp 71-76

55. Gliese U, Ngrskov S, Nielsen TN (1996) Chromatic dispersion in fiber-optic microwave and millimeter-wave links. IEEE Trans Microwave Theor Tech 44(10):1716-1724

56. Yang Y, Lim C, Nirmalathas A (2010) Multichannel digitized RFover-Fiber transmission based on bandpass sampling and FPGA. IEEE Trans Microwave Theor Tech 58(11):3181-3188

57. Yang Y, Lim C, Nirmalathas A (2011) Comparison of energy consumption of integrated optical wireless access networks. In: Optical Fibre Communications (OFC 2011), paper JWA82

58. Clark TR, Dennis ML (2007) Coherent optical phase-modulation link. IEEE Photonics Technol Lett 19(16):1206-1208

59. Gonzalez NG, Zibar D, Caballero A, Monroy IT (2010) Experimental 2.5-Gb/s QPSK WDM phase-modulated radio-over-fiber link with digital demodulation by a k-means algorithm. IEEE Photonics Technol Lett 22(5):335-337

60. Caballero A, Zibar D, Sambaraju R, Martí J, Monroy IT (2012) High-capacity $60 \mathrm{GHz}$ and $75110 \mathrm{GHz}$ band links employing all-optical OFDM generation and digital coherent detection. J Lightwave Technol 30(1):147-155

61. Leven A, Kaneda N, Corteselli S (2010) Real-time implementation of digital signal processing for coherent optical digital communication systems. IEEE J Sel Areas Quantum Electron 16(5):1227-1234

62. Savory SJ (2010) Digital coherent optical receivers: algorithms and subsystems. IEEE J Sel Areas Quantum Electron 16(5):11641170

63. Kalantari-Sabet B, Mieku M, Gomes NJ, Mitchell JE (2008) Performance impairments in single-mode Radio-Over-Fiber systems due to MAC constraints. J Lightwave Technol 26(15):25402548

64. Rideout H, Seregelyi J, Yao JP (2007) A true-time delay beamforming system incorporating a wavelength tunable optical phaselock loop. J Lightwave Technol 25(7):1761-1769

65. Pozzi F, Rue RMDL (2006) Dual-wavelength InAlGaAsInP laterally coupled distributed feedback laser. J Opt Commun Netw 1(4):324-330 